\title{
Ultrasonographic Evaluation of Therapeutic Effects of Complex Decongestive Therapy in Breast Cancer-Related Lymphedema
}

\author{
Jae Hyun Lee, MD, Bae Wook Shin, MD, Ho Joong Jeong, MD,
} Ghi Chan Kim, MD, Dong Kyu Kim, MD, Young-Joo Sim, MD

Department of Physical Medicine and Rehabilitation, Kosin University College of Medicine, Busan, Korea

\begin{abstract}
Objective To evaluate the usefulness of ultrasonography as a follow-up tool for evaluating the effects of complex decongestive physiotherapy (CDPT) in breast cancer-related lymphedema (BCRL).

Methods Twenty patients with BCRL were enrolled in this study. All patients had undergone therapy in the CDPT program for 2 weeks. Soft tissue thickness of both the affected and unaffected upper limb was measured before and after CDPT. The measurements were taken at 3 points (the mid-point between the medial and lateral epicondyles at the elbow level, $10 \mathrm{~cm}$ proximal and $10 \mathrm{~cm}$ distal to the elbow) with and without pressure. We then calculated the compliance of soft tissue before and after CDPT. Circumferences of both the affected and unaffected upper limb were also measured before and after CDPT at the 3 defined points.

Results After 2 weeks of the CDPT program, the circumference and soft tissue thickness of the unaffected upper limb did not significantly change. In the affected upper limb, the circumference was significantly reduced in the 3 point, when compared with measurements taken prior to treatment. Additionally, soft tissue thickness was significantly reduced at the elbow and $10 \mathrm{~cm}$ proximal to the elbow. After CDPT, compliance at each of the 3 points had increased, but this trend was not significantly different.

Conclusion Our results showed that arm circumference and ultrasonography-derived soft tissue thickness was useful as a way of assessing therapeutic effects of CDPT.
\end{abstract}

Keywords Lymphedema, Ultrasonography, Complex decongestive physiotherapy

Received May 10, 2013; Accepted July 9, 2013

Corresponding author: Young-Joo Sim

Department of Physical Medicine and Rehabilitation, Kosin University College of Medicine, 262 Gamcheon-ro, Seo-gu, Busan 602-702, Korea Tel: +82-51-990-6261, Fax: +82-51-241-2019, E-mail: oggum@daum.net

(c) This is an open-access article distributed under the terms of the Creative Commons Attribution Non-Commercial License (http://creativecommons. org/licenses/by-nc/3.0) which permits unrestricted noncommercial use, distribution, and reproduction in any medium, provided the original work is properly cited.

Copyright $\odot 2013$ by Korean Academy of Rehabilitation Medicine

\section{INTRODUCTION}

Lymphedema is a disease caused by accumulating lymph fluid in the skin and subcutaneous tissue due to lymphatic circulatory disturbances. Depending on the underlying cause, it can be divided into primary and secondary lymphedema. Primary lymphedema is a congenital disease caused by lymphatic hypoplasia, while secondary lymphedema is acquired, usually due to malignancy, radiation therapy, surgery, injury, or infection 
$[1,2]$. Breast cancer-related lymphedema (BCRL) is a secondary lymphedema resulting from surgical resection of axillary lymph nodes, radiation-induced fibrosis, obstruction of lymphatics by metastatic tumor, and/or infection [3].

Generally, complex decongestive physiotherapy (CDPT), pneumatic compression, and compression stockings are used to treat BCRL. Among these methods, CDPT is the most effective conservative therapy and consists of manual lymphatic drainage, non-elastic bandage compression therapy, exercise, and skin care [4].

When diagnosing lymphedema, a patient's history and physical examination are the most important factors. Upper extremity circumference measurements, water displacement volumetry, and infrared optoelectronic volumetry can be used for lymphedema volume measurement $[5,6]$. To examine the anatomical characteristics of the involved upper extremity, computed tomography (CT), magnetic resonance imaging (MRI), and ultrasonography can be used [5-9]. In addition, lymphoscintigraphy can be used to further characterize the function and anatomy of the lymphatic system [10].

Recent research has mainly focused on the use of ultrasonography in the diagnosis of lymphedema. Ultrasonography is a relatively inexpensive method to observe the soft-tissue characteristics. Kim et al. [9] reported that lymphedema can reliably be diagnosed with ultrasonography by comparing the thickness of skin and subcutaneous tissue and also evaluating the compliance of subcutaneous tissue in clinical settings. In addition, ultrasonography is less expensive than CT or MRI, easily accessible, safe from radiation exposure, and noninvasive, which means that it can be used as a diagnostic tool for lymphedema and also as a method to determine treatment efficacy. In spite of these benefits, studies on ultrasonography for lymphedema have focused on diagnosis but not for evaluation of therapeutic intervention. This study aimed to determine not only the usefulness of ultrasonography in elucidating the effects of CDPT in patients with lymphedema after breast cancer surgery, but also whether ultrasonography has a clinical significance as a follow-up test in confirming treatment efficacy.

\section{MATERIALS AND METHODS}

\section{Subjects}

This study recruited patients who had breast cancer surgery between February 2010 and November 2012 and had experienced subsequent lymphedema. The diagnosis of lymphedema was defined by the following criteria: 1) a difference in circumference between the affected and unaffected upper extremities of more than $2 \mathrm{~cm}$; and 2) lymphoscintigraphy demonstrating slow or no lymphatic circulation compared to the unaffected side, dermal back flow, deep lymphatics, and collateral circulation [8]. Patients were excluded according to the following criteria: 1) patients with edema before breast cancer treatment; 2) medical history including other causes of edema, such as heart, kidney, or liver diseases; and 3) recurrence or metastasis of breast cancer diagnosed in follow-up imaging studies, such as positron emission tomography-CT, CT, or MRI.

Twenty-three patients with first admission for lymphedema treatment were enrolled after given written informed consent. During the lymphedema treatment, 2 patients were diagnosed with recurrence of ipsilateral breast cancer, and 1 patient had died with bilateral lung metastasis. Therefore, these 3 patients were dropped from the analysis. In total, 20 patients were included in the final analysis.

\section{Treatment method}

All 20 patients underwent a standard protocol of CDPT (manual lymphatic drainage, non-elastic bandage compression therapy, exercise, and skin care). The CDPT was performed by a trained physical therapist for 1 hour a day, 5 days a week for 2 weeks, with methods identical to those published in previous studies $[4,10]$. The manual drainage was performed in a proximal to distal direction with light skin massage, it was not stroking. Non-elastic bandages were applied and changed twice daily. All patients were also educated on appropriate skin care, such as avoiding extreme heat, proper skin hygiene, avoiding trauma, and applying moisturizer daily. The patients were also provided with an individualized exercise program, such as abdominal breathing exercise and neck and shoulder stretching exercises, to help facilitate lymphatic flow and improve range of motion.

\section{Measurement of therapeutic effects Measurement sites}

In both affected and unaffected upper extremities, circumference, soft tissue thickness, and compliance were measured at three points. The 3 points were defined as 
follows: 1) elbow, the mid-point between the medial and lateral epicondyles; 2) proximal, $10 \mathrm{~cm}$ proximal to the elbow point along the line of the humerus and the bicipital groove between the mid-point of the medial and lateral epicondyles; and 3) distal, $10 \mathrm{~cm}$ distal to the elbow point along the line of the radial and ulnar styloid processes between the mid-point of the medial and lateral epicondyles (Fig. 1). Compliance was additionally measured in the most edematous area (hardest point), as subjectively reported by each patient.

\section{Circumference measurement}

In both upper limbs, circumferences were measured with a tape measure at the 3 points described above. During each measurement, patients were seated with their forearm supinated and relaxed on a pillow. These measurements were obtained before and after CDPT.

\section{Soft tissue thickness and compliance measurement by} ultrasonography

Soft tissue thickness and compliance were measured for the 3 points as described above, before and after CDPT. Additionally, we also measured compliance at the

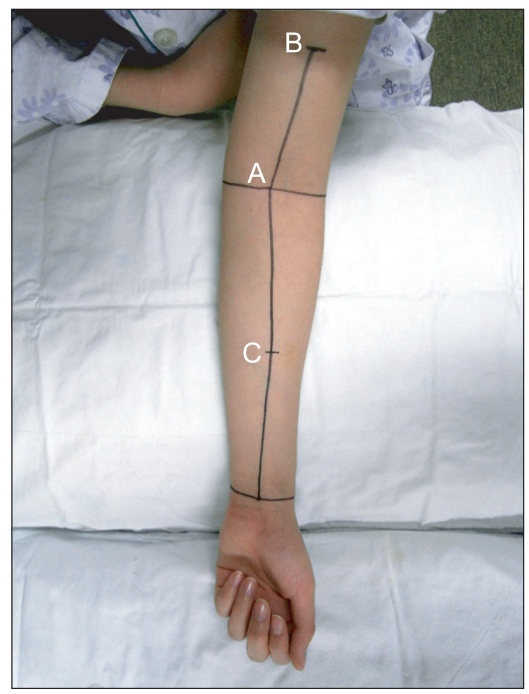

Fig. 1. A photograph illustrating the measurement sites on a patient's upper limb. Mid-point of the wrist crease, the mid-point between the medial and lateral epicondyles at the level of the elbow, and the bicipital groove were marked. These three points were connected linearly, and the 3 measuring sites were marked: (A) the midpoint at the elbow (elbow), (B) above the mid-point at the elbow level (proximal), and (C) below the mid-point at the elbow level (distal). hardest territory of 6 territories in affected upper limb. During each ultrasonographic evaluation, patients were instructed to keep the same position as for the circumference measurement. Soft tissue thickness was defined the sum of skin and subcutaneous tissue thicknesses on ultrasonography (Fig. 2). No pressure was applied during ultrasonographic measurements. For compliance measurements, soft tissue thickness was measured once again while applying maximal compression, which was defined as compression with the pressure at which additional compression did not produce a notable decrease in the thickness of the soft tissue. Based on the study by Kim et al. [9], we defined compliance as the difference in soft tissue thickness with and without compression divided by soft tissue thickness without compression.
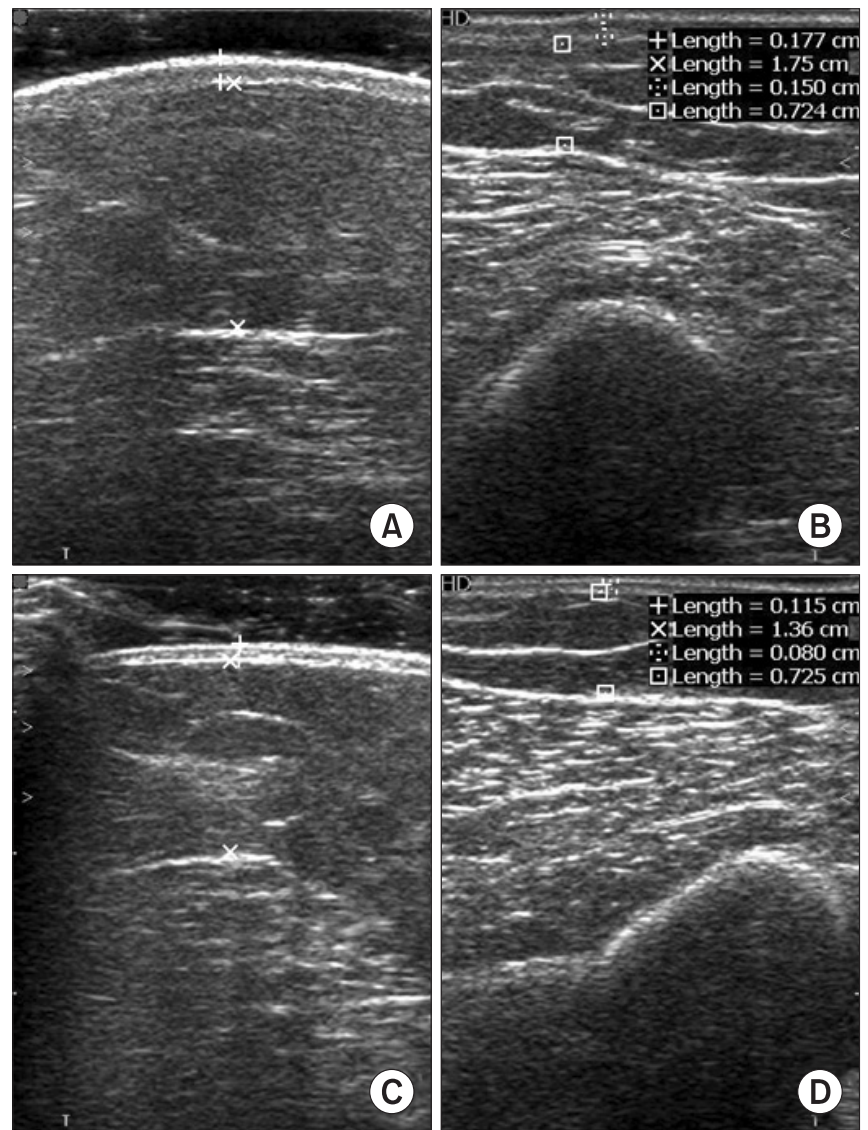

Fig. 2. Ultrasonographic images measuring below the mid-point at the elbow level on both upper limbs. (A) Affected side without compression, (B) affected side with compression, (C) unaffected side without compression, and (D) unaffected side with compression.

www.e-arm.org 
Thickness without pressure-

Compliance $=\frac{\text { Thickness with maximal pressure }}{\text { Thickness without pressure }}$

\section{Statistical analysis}

SPSS ver. 12.0 (SPSS Inc., Chicago, IL, USA) for Windows was used for statistical analysis, and the paired t-test was

Table 1. Characteristics of subjects

\begin{tabular}{|c|c|}
\hline Characteristic & Value \\
\hline Age (yr) & $49.72 \pm 7.84$ \\
\hline Body mass index & $24.25 \pm 2.56$ \\
\hline \multicolumn{2}{|l|}{ Miller stage } \\
\hline I & 6 \\
\hline II & 10 \\
\hline III & 4 \\
\hline \multicolumn{2}{|l|}{ Hardest area } \\
\hline Forearm & $14(70)$ \\
\hline Upper arm & $6(30)$ \\
\hline \multicolumn{2}{|l|}{ Lymphedema side } \\
\hline Right & $10(50)$ \\
\hline Left & $10(40)$ \\
\hline \multicolumn{2}{|l|}{ Operation } \\
\hline Modified radical & $15(75)$ \\
\hline Partial & $3(15)$ \\
\hline None & $2(10)$ \\
\hline Radiotherapy & $15(75)$ \\
\hline Chemotherapy & $17(85)$ \\
\hline
\end{tabular}

Values are presented as mean \pm standard deviation or number (\%). used to analyze the circumference and thickness of soft tissue before and after CDPT. Statistical significance was set at $\mathrm{p}<0.05$.

For the calculation of the sample size, we used GPower (ver. 3.1.7) for Windows with an effect size of 0.5 , an alpha risk of 0.05 , and a power of 0.70 . The calculation of the sample size was 21 patients. Considering a $10 \%$ dropout rate in each group, the corrected sample size was 23 patients.

\section{RESULTS}

\section{Characteristics}

The twenty subjects were all women with an average age of $49.72 \pm 7.84$ years. The average body mass index (BMI) was 24.25 \pm 2.56 . Fourteen of the patients reported that the hardest area was in the forearm (70\%), and the remaining six patients noted it to be in the upper arm (30\%) (Table 1). Thirteen out of the 14 patients also reported significant hardness in the ulnar forearm area, and the remaining patient complained of edema in the radial forearm. All of the 6 patients who noted the hardest area in upper arm reported significant hardness in the medial upper arm area. Soft tissue thickness and compliance were not associated with demographic or clinical characteristics $(\mathrm{p}>0.05)$.

\section{Therapeutic effects of CDPT based on circumference changes}

In the affected upper extremities, the mean pre-treatment circumferences were $25.24 \pm 2.32,30.25 \pm 3.25$, and $27.30 \pm 1.95 \mathrm{~cm}$ at the distal, proximal, and elbow points,

Table 2. Comparison of limb circumference pre- and post-treatment

\begin{tabular}{|c|c|c|c|}
\hline & \multicolumn{2}{|c|}{ Circumference (cm) } & \multirow{2}{*}{ p-value } \\
\hline & Pre & Post & \\
\hline \multicolumn{4}{|l|}{ Unaffected upper limb } \\
\hline $10 \mathrm{~cm}$ distal to elbow & $23.18 \pm 2.10$ & $23.09 \pm 1.92$ & 0.25 \\
\hline Elbow & $24.94 \pm 1.82$ & $24.83 \pm 1.88$ & 0.08 \\
\hline $10 \mathrm{~cm}$ proximal to elbow & $27.76 \pm 2.71$ & $27.67 \pm 2.57$ & 0.29 \\
\hline \multicolumn{4}{|l|}{ Affected upper limb } \\
\hline $10 \mathrm{~cm}$ distal to elbow & $25.24 \pm 2.32$ & $24.49 \pm 2.23$ & $0.00^{*}$ \\
\hline Elbow & $27.30 \pm 1.95$ & $25.81 \pm 1.85$ & $0.00^{*}$ \\
\hline $10 \mathrm{~cm}$ proximal to elbow & $30.25 \pm 3.25$ & $28.99 \pm 3.15$ & $0.00^{*}$ \\
\hline
\end{tabular}

Values are presented as mean \pm standard deviation.

${ }^{*} \mathrm{p}<0.05$, according to paired t-test between pre- and post-treatment measurements in each group. 
respectively. The mean post-treatment circumferences were $24.49 \pm 2.23,28.99 \pm 3.15$, and $25.81 \pm 1.85 \mathrm{~cm}$, in the same respective locations. In all 3 points, the circumferences had significantly decreased after CDPT $(\mathrm{p}<0.01$ in 3 points) (Table 2).

\section{Therapeutic effects of CDPT based on soft tissue thickness changes}

In the affected upper extremity, the pre-CDPT mean soft tissue thickness measurements were $0.82 \pm 0.31$, $0.92 \pm 0.40$, and $0.67 \pm 0.23 \mathrm{~cm}$ at the distal, proximal, and elbow points, respectively. After CDPT, the respective values were $0.80 \pm 0.27,0.80 \pm 0.25$, and $0.56 \pm 0.17 \mathrm{~cm}$. Soft tissue thicknesses of the elbow $(\mathrm{p}=0.00)$ and proximal points $(\mathrm{p}=0.01)$ had significantly decreased after treatment (Table 3).
Therapeutic effects of CDPT based on soft tissue compliance changes

In the unaffected upper extremity, compliance measured at the elbow, proximal, and distal points did not significantly change before and after treatment (Table 4). In the affected upper extremity, compliance was not significantly different between pre- and post-treatment assessments (Table 4). Additionally, compliance measured at the hardest territory did not significantly change with treatment.

\section{DISCUSSION}

Lymphedema is the most common complication of breast cancer, causing disruption of lymphatic circulation due to injuries to lymphatic vessels. An excessive amount of protein-rich lymphatic fluid accumulates in

Table 3. Comparison of soft tissue thickness pre- and post-treatment

\begin{tabular}{|c|c|c|c|}
\hline & \multicolumn{2}{|c|}{ Soft tissue thickness $(\mathrm{cm})$} & \multirow{2}{*}{ p-value } \\
\hline & Pre & Post & \\
\hline \multicolumn{4}{|l|}{ Unaffected upper limb } \\
\hline $10 \mathrm{~cm}$ distal to elbow & $0.69 \pm 0.26$ & $0.68 \pm 0.27$ & 0.25 \\
\hline Elbow & $0.53 \pm 0.15$ & $0.50 \pm 0.15$ & 0.06 \\
\hline $10 \mathrm{~cm}$ proximal to elbow & $0.81 \pm 0.34$ & $0.75 \pm 0.36$ & 0.07 \\
\hline \multicolumn{4}{|l|}{ Affected upper limb } \\
\hline $10 \mathrm{~cm}$ distal to elbow & $0.82 \pm 0.31$ & $0.80 \pm 0.27$ & 0.65 \\
\hline Elbow & $0.67 \pm 0.23$ & $0.56 \pm 0.17$ & $0.00^{*}$ \\
\hline $10 \mathrm{~cm}$ proximal to elbow & $0.92 \pm 0.40$ & $0.80 \pm 0.25$ & $0.01^{*}$ \\
\hline
\end{tabular}

Values are presented as mean \pm standard deviation.

${ }^{*} \mathrm{p}<0.05$, according to paired t-test between pre- and post-treatment measurements in each group.

Table 4. Comparison of tissue compliance pre- and post-treatment

\begin{tabular}{lccc}
\hline & \multicolumn{2}{c}{ Compliance } & \multirow{2}{*}{ p-value } \\
\cline { 2 - 3 } & & Post & \\
\hline Unaffected upper limb & & & 0.55 \\
\hline $10 \mathrm{~cm}$ Distal to elbow & $0.20 \pm 0.15$ & $0.22 \pm 0.17$ & 0.47 \\
\hline Elbow & $0.32 \pm 0.17$ & $0.26 \pm 0.24$ & 0.08 \\
\hline Affected upper limb & $0.53 \pm 0.16$ & $0.50 \pm 0.16$ & \\
\hline $10 \mathrm{~cm}$ Distal to elbow & & & 0.79 \\
\hline Elbow & $0.18 \pm 0.17$ & $0.18 \pm 0.13$ & 0.62 \\
\hline $10 \mathrm{~cm}$ Proximal to elbow & $0.35 \pm 0.19$ & $0.38 \pm 0.18$ & 0.40 \\
\hline
\end{tabular}

Values are presented as mean \pm standard deviation. 
the interstitium, and this accumulation increases the activities of neutrophils, macrophages, and fibroblasts, which stimulate tissue fibrosis and thickening of soft tissues [10]. Subsequently, these changes alter the physiological characteristics of soft tissue, including compliance and thickness [8]. CDPT, a conventional treatment for lymphedema, promotes lymphatic flow through the functioning capillary lymphatic vessels and activates collateral circulation. It leads to increased emission of fluid and proteins, softens fibrotic tissues, and improves the histologic changes associated with lymphedema. Consequently, the volume of lymphedema and risk of infection decrease with CDPT [11].

Lymphedema is a disease that is characterized not only by fluid accumulation in the interstitium but also by histologic changes. Although it is necessary to obtain histological information to judge the efficacy of treatment, simple circumference and volume measurements are commonly used for diagnosis and follow-up evaluation because imaging studies, such as CT and MRI are both expensive and time-consuming. Thus, the effects of CDPT have been difficult to examine on a histological level. In this study, pre- and post-treatment circumference measurements were compared in patients with BCRL. These circumference measurements were significantly decreased, confirming the significant effect of CDPT which had been previously published. After 2 weeks of CDPT, soft tissue thickness at the elbow and proximal points measured by ultrasonography showed a significant decrease. However, compliance did not show any significant changes after treatment at any of the 3 points. And soft tissue thickness and compliance were not associated with demographic or clinical characteristics the patients in this study. These results were similar to previous studies that reported no correlations between demographic characteristics, such as BMI or age and compliance, in concordance with this study [6].

In a previous study, Kim et al. [9] had recruited 13 healthy participants as subjects and measured the thickness and compliance of all soft tissue including skin and subcutaneous tissue using ultrasonography. The interand intra-rater correlation coefficients of the subcutaneous tissue thickness and total soft tissue measurements were more than 0.75 , which indicates excellent reliability [9]. However, the intra-rater correlation coefficient was lower than 0.75 for skin thickness measurement; thus, the subcutaneous tissue and total soft tissue thickness measurements had higher reliability than that of skin thickness measurement [9]. Based on these results, this study tried to observe the effects of CDPT by measuring total soft tissue thickness and compliance using ultrasonography. In diagnosing lymphedema, some studies reported that the thickness of skin, subcutaneous skin, and total soft tissue measurements of the affected upper extremity determined were higher than those measurements on the unaffected side, which are findings our study results reflect $[2,8,11]$. However, these previous studies have only compared the affected and unaffected upper extremities of the patients with lymphedema. In this study, these measurements were used to assess the changes that occur to the upper extremities in patients before and after CDPT. Ultrasonography measurements have revealed that CDPT was effective in decreasing softtissue thickness.

Lymphedema can alter the resistance to pressure and elasticity of skin and subcutaneous tissues, which is related to a patient's perception of hardness in the upper extremity afflicted with lymphedema [11]. The resistance to pressure is calculated as the difference between soft tissue thicknesses measured without pressure and with maximal pressure, which is similar to the concept of compliance [8]. In this study, this difference was divided by the soft tissue thickness measured without pressure, and this ratio was defined as compliance to reduce errors related to typical variation in soft tissue thickness between patients. In previous studies, compliance of lymphedematous upper extremity was reported to be lower than that of the unaffected side, suggesting that compliance measured by ultrasonography in the upper extremity could a useful measure of lymphedema [8]. We expected changes in compliance due to reduced fibrosis of the affected arm after CDPT. Two weeks of CDPT improved most subjective complaints of hardness and heaviness in the upper extremity, but compliances measured by ultrasonography in the soft tissue was not significantly different. Despite the idea that tissue fibrosis improves with CDPT, the changes in thicknesses under pressure were limited due to the overall decrease in thickness observed after CDPT. Additionally, 15 out of 20 subjects had lymphedema of stage 2 or higher, meaning that tissue fibrosis was more severe relative to the respective volume increase. Thus, the effects of CDPT were not marked, and histological changes might not have been evident. In the end, whether the compliance as measured 
by ultrasonography can accurately reflect histological characteristics is uncertain.

This study found that using ultrasonography to measure the thickness of soft tissues before and after CDPT can be a useful method in patients with BCRL. Ultrasonography examination is inexpensive, easily accessible, and can demonstrate histological changes, which cannot be evaluated by circumference measurement. During the ultrasonographic evaluation of lymphedema, various musculoskeletal diseases that occur after breast cancer surgery can be screened for. Therefore, using ultrasonography to observe efficacy of CDPT can be clinically useful. However, compliance measurements by ultrasonography did not provide clear correlations with treatment outcomes for lymphedema. More studies are need to take into account the stage, degree, and histological characteristics of lymphedema.

The main limitations of this study were its small sample size and the fact that most of the patients included had stage 2-3 chronic lymphedema. These limitations do not allow generalization of the study results to patients with less severe lymphedema. Moreover, ultrasonography measurements are operator dependent, and technical differences in pressure application during subcutaneous volume and compliance measurements may have affected the results. Further long-term studies with larger sample sizes are needed to verify the usefulness of ultrasonography as a tool for evaluating the therapeutic effect of CDPT.

In this study, 2 weeks of CDPT significantly decreased the thickness of lymphedematous soft tissues in patients with BCRL, as measured by ultrasonography, but did not affect the compliances measured. Ultrasonography can be a useful indicator of treatment efficacy for lymphedema, but further studies with larger sample sizes are needed to examine its accuracy in assessing tissue compliance.

\section{CONFLICT OF INTEREST}

No potential conflict of interest relevant to this article was reported.

\section{ACKNOWLEDGMENTS}

This study was supported by a grant from Kosin Univer- sity College of Medicine in 2011.

\section{REFERENCES}

1. Jeong HJ, Sim YJ, Hwang KH, Kim GC. Cause of shoulder pain in women with breast cancer-related lymphedema: a pilot study. Yonsei Med J 2011;52:661-7.

2. Han NM, Cho YJ, Hwang JS, Kim HD, Cho GY. Usefulness of ultrasound examination in evaluation of breast cancer-related lymphedema. J Korean Acad or Rehabil Med 2011;35:101-9.

3. Petrek JA, Pressman PI, Smith RA. Lymphedema: current issues in research and management. CA Cancer J Clin 2000;50:292-307.

4. Hwang JH, Choi JY, Lee JY, Hyun SH, Choi Y, Choe YS, et al. Lymphscintigraphy predicts response to complex physical therapy in patients with early stage extremity lymphedema. Lymphology 2007;40:172-6.

5. Deltombe T, Jamart J, Recloux S, Legrand C, Vandenbroeck N, Theys S, et al. Reliability and limits of agreement of circumferential, water displacement, and optoelectronic volumetry in the measurement of upper limb lymphedema. Lymphology 2007;40:26-34.

6. Tomczak H, Nyka W, Lass P. Lymphoedema: lymphoscintigraphy versus other diagnostic techniques: a clinician's point of view. Nucl Med Rev Cent East Eur 2005;8:37-43.

7. Stanton AW, Badger C, Sitzia J. Non-invasive assessment of the lymphedematous limb. Lymphology 2000;33:122-35.

8. Lim CY, Seo HG, Kim K, Chung SG, Seo KS. Measurement of lymphedema using ultrasonography with the compression method. Lymphology 2011;44:72-81.

9. Kim W, Chung SG, Kim TW, Seo KS. Measurement of soft tissue compliance with pressure using ultrasonography. Lymphology 2008;41:167-77.

10. Hwang JH, Lee KW, Kwon JY, Kim BT, Choi JY, Lee BB, et al. Improvement of lymphatic function after complex physical therapy change of lymphoscintigraphy. J Korean Acad Rehabil Med 1998;22:698-704.

11. Tassenoy A, De Mey J, De Ridder F, Van Schuerbeeck P, Vanderhasselt T, Lamote J, et al. Postmastectomy lymphoedema: different patterns of fluid distribution visualised by ultrasound imaging compared with magnetic resonance imaging. Physiotherapy 2011;97:234-43. 